\title{
Extracting the thermal SZ signal from heterogeneous mil- limetre data sets
}

\author{
H. Bourdin ${ }^{1, *}$, A.S. Baldi ${ }^{1}, A$. Kozmanyan $^{1}$, and P. Mazzotta ${ }^{1}$ \\ ${ }^{1}$ Università degli studi di Roma 'Tor Vergata'; Via della ricerca scientifica, 1; 00133 Roma (Italy)
}

\begin{abstract}
Complementarily to X-ray observations, the thermal SZ effect is a powerful tool to probe the baryonic content of galaxy clusters from their core to their peripheries. While contaminations by astrophysical and instrumental backgrounds require us to scan the thermal SZ signal across various frequencies, the multi-scale nature of cluster morphologies require us to observe such objects at various angular resolutions. We developed component separation algorithms that take advantage of sparse representations to combine these heterogeneous pieces of information, separate the thermal SZ signal from its contaminants, detect and map the thermal SZ signal of galaxy clusters from nearby to more distant clusters of the Planck catalogue. Spatially weighted likelihoods allow us in particular to connect parametric fittings of the component Spectral Energy Distribution with wavelet and curvelet imaging, but also to combine signals registered with beams of various width. Such techniques already allow us to detect sub-structures in the peripheries of nearby clusters with Planck, and could be extended to observations performed at higher angular resolutions.
\end{abstract}

\section{Introduction}

Complementarily with X-ray emission, the thermal Sunyaev-Zel'dovich (tSZ) signal is a powerful tracer of the hot gas content of galaxy clusters, thus of the deep gravitational potential well that these hot atmospheres fill in. Due to the quadratic and linear dependences of X-ray surface brightness and millimetre $(\mathrm{mm})$ optical depth of clusters with their hot gas density, X-ray observations mostly enlighten the innermost cluster regions while $\mathrm{mm}$ observations reveal us more peripheral regions where the cosmic matter continuously accretes and virialises. Combining these signals also allows us to probe the helium abundance in the Intra-Cluster Medium (ICM) or to measure the intrinsic sizes of clusters, thus to infer the cosmological parameters that underpin their angular diameter distance. The redshift independence of the tSZ signal further helps us to probe cosmology with cluster counts, in particular via the build-up of nearly mass-selected cluster catalogues from wide mm sky surveys.

The tSZ signal is a characteristic spectral distortion of the CMB spectrum observable in a frequency range of about 50 to $500 \mathrm{GHz}$. Except for the exceptionally dense central regions of spatially resolved clusters, the intensity of this distortion competes with localised intensity variations of other diffuse or compact astrophysical sources, including anisotropies of the Cosmic Microwave Background (CMB) or (extra)-Galactic thermal dust emission.

\footnotetext{
*e-mail: herve.bourdin@roma2.infn.it
} 


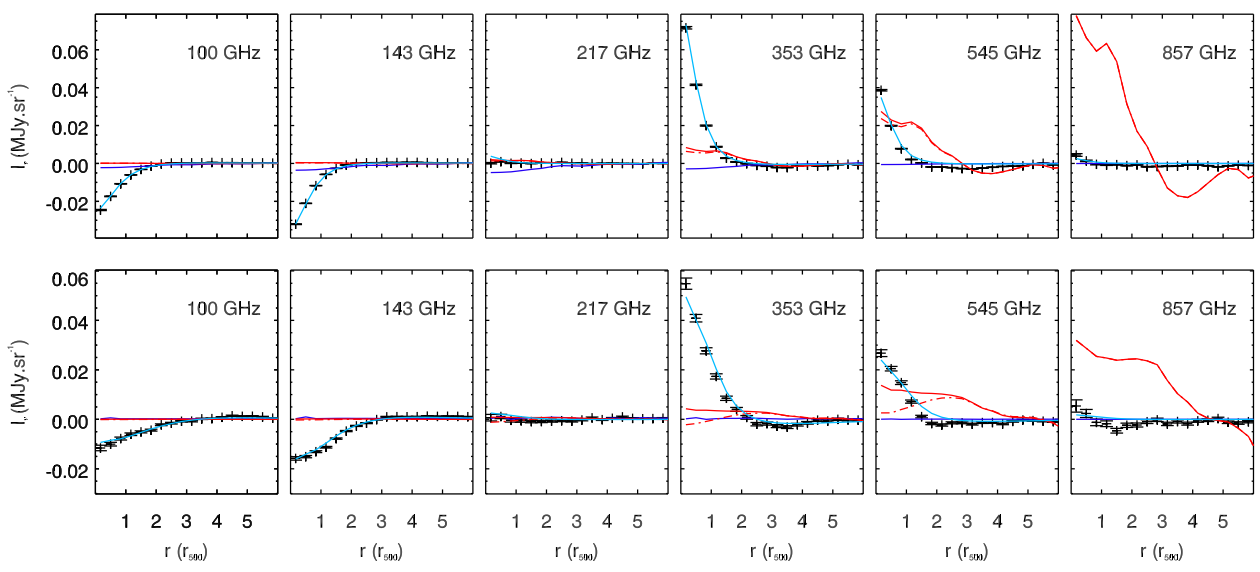

Figure 1. Top panel: Stacked tSZ signal detected by the Planck High Frequency Instrument (HFI) towards 61 nearby SZ sources $(z<0.5)$ of the Planck catalogue. Bottom panel: Same as top panel as for 23 distant ( $\mathrm{z}>0.5$ ) clusters. HFI maps are corrected for the sum of the anisotropies of the CMB (dark blue curve) and (extra)-Galactic thermal dust emission (red curves). Dot-dashed and continuous red curves depict thermal dust anisotropies that have been fitted assuming two components (Galactic) and three components (Galactic + intra-cluster) SEDs, respectively. Light blue line depicts the arithmetic mean of tSZ templates that have been fitted to the data set for each galaxy cluster.

Therefore the tSZ signal is best decontaminated and restored via the combination of observations performed at various frequencies that cover its spectral signature. The multi-scale nature of cluster morphologies also requires us to combine observations that gather various spatial frequencies, typically corresponding to spatial scales in a range of few arcseconds to several arcminutes. For these reasons, tSZ measurements often result from the combination of heterogeneous data sets gathered by spatial and ground based experiments, characterised by their specific frequency responses, beams, noise contamination of instrumental or atmospheric origin.

Focalised on analyses of frequency maps of the Planck High Frequency Instrument (HFI), this report illustrates a few strategies that combine sparse image denoising and spatially resolved spectroscopy in order to demix the diffuse components that contamine the tSZ signal. Section 2 will show that radial profiles of the Compton parameter of nearby or distant $(z>0.5)$ galaxy clusters can be extracted via a simple linear combination of wavelet denoised frequency maps and underlying components. Section 3 and 4 will be devoted to Compton parameter spectral-imaging around nearby galaxy clusters and across the sky, respectively.

\section{SZ photometry}

Assuming a self-similar shape of cluster atmospheres, the Planck collaboration used detection algorithms (e.g. matched filters) to generate a catalogue of about 1200 confirmed clusters [1]. Beyond cluster detections, accurate measurements of the cluster fluxes require us to improve our knowledge of the individual pressure profiles of clusters, and to model the diffuse instrumental and astrophysical backgrounds that contaminate the tSZ cluster signal. By covering the tSZ signal in both its decrement and increment parts $(v<217$ and $v>217 \mathrm{GHz}$, respectively) and including two bands (217 and $857 \mathrm{GHz}$ ) where the tSZ signal is negligible 
with respect to the noise level, the Planck HFI has been especially designed to separate the tSZ signal form its contaminants.

Taking advantage from this design, we constructed an algorithm that allows us to extract pressure profiles of clusters in the Planck catalogue [2]. Following this algorithm, the 217 and $857 \mathrm{GHz}$ maps are denoised via a wavelet filtering and combined with one another to model spatial variations of the CMB and Galactic thermal dust. All HFI frequency maps are further used to fit the Spectral Energy Distribution (SED) of the Galactic thermal dust template outside each individual cluster ${ }^{1}$. Subtracting CMB and thermal dust anisotropies from each high-pass filtered frequency map thus provides us with a residual that can be spatially and spectrally fit with a tSZ template. Using this approach for two subsamples of the Planck catalogue of SZ sources [1] separated by $z=0.5$, we jointly fitted analytical cluster pressure profiles to such templates and to X-ray surface brightness and spectroscopic temperature profiles extracted from XMM-Newton and Chandra data. We recovered pressure profiles that exhibit a tight self-similarity in the innermost cluster regions $\left(r \leq r_{500}\right)$ and a larger dispersion in the peripheries $\left(r>r_{500}\right)$. As expected from theoretical models of massive cluster growth, stacked pressure profile shapes did not show any evolution between the two redshift intervals. An additional outcome of this analysis is the thermal dust template that we jointly fitted with each tSZ template. Interestingly, the SED of the Galactic thermal dust template that we fitted outside galaxy clusters needed to be parametrised by two grey-body components in order to fit the data set within the overall frequency range of the HFI (see also [3]). After removal of the Galactic thermal dust template, a thermal dust excess of intra-cluster origin could also be detected and modelled for distant clusters in our sample. As suggested by analyses of the stacked tSZ signal of distant clusters by the Planck collaboration [4], we used a redshifted version of the Galactic thermal dust SED for this purpose. The spatial extension and SED of intra-cluster thermal dust should be further investigated by combining Planck data with observations of distant clusters performed at higher angular resolutions.

\section{Cluster imaging}

Looking for substructures inside and around galaxy clusters requires us to combine all spatial frequencies that contribute to the tSZ signal, and to look for highly anisotropic details such as filaments. As shown on mock observations of interacting clusters with planck HFI [5], the tSZ signal can be separated from CMB and Galactic thermal dust anisotropies through the use of wavelet and curvelet transforms of these components. Let us introduce frequency dependent templates that combine SEDs of these components, $\mathrm{f}_{\text {comp }}(v)$, normalised with amplitudes, $\mathrm{A}_{\text {comp }}(k, l)$, corrected for an instrument response, $R(v)$. These templates, $y(k, l, v)$, read as a function of spatial coordinates $k, l$ and frequency $v$ as:

$$
y(k, l, v)=R(v) \times\left[\mathrm{A}_{\mathrm{SZ}}(k, l) \mathrm{f}_{\mathrm{SZ}}(v)+\mathrm{A}_{\text {dust }}(k, l) \mathrm{f}_{\mathrm{dust}}(v)+\mathrm{A}_{\mathrm{CMB}}(k, l)\right] .
$$

Introducing frequency maps $I(k, l, v)$, and variance maps $\sigma(k, l, v)^{2}$, wavelet coefficients, $\mathscr{W}(k, l, a)$, that encode the spatial variations of each component amplitude, $\mathbf{A}(k, l)$, at scale $a$, are computed from the combinations of two likelihood maximisations that we spatially weight by the negative and the positive part of a B3-spline wavelet (see e.g. [6]), $\Psi_{+}$and $\Psi_{-}$:

$$
\chi_{\Psi_{+,-}}^{2}(k, l, a)=\sum_{i, j, v} \Psi_{+,-}(i, j, a) \frac{[I(i-k, j-l, v)-y(i-k, j-l, v)]^{2}}{\sigma(i-k, j-l, v)^{2}} ;
$$

\footnotetext{
${ }^{1}$ Typically in a radii range of $5 \times r_{500}<r<12 \times r_{500}$, where $r_{500}$ is the radius of a sphere encompassing a matter over-density whose average value equals 500 times the critical density of the Universe at the cluster redshift.
} 


$$
\left.\mathscr{W}(k, l, a)=\frac{1}{2} \times \underset{\mathbf{A}}{\arg \min }\left(\chi_{\Psi_{+}}^{2}(k, l, a)\right)-\underset{\mathbf{A}}{\arg \min }\left(\chi_{\Psi_{-}}^{2}(k, l, a)\right)\right] .
$$

In the same way, the variance of the wavelet coefficients can be computed using a generalised Fisher information that derives from the same combination of likelihood maximisations. Such coefficients subsequently allow us to compute wavelet transforms to prevalently analyse isotropic details of the CMB maps and curvelet transforms to detect edges within maps of the tSZ and Galactic thermal dust signals. A thresholding of component coefficients whose amplitude exceeds the noise level allows us to define a sparse basis that we use as a support for denoising of the CMB, thermal dust and tSZ signals. Additional non-linear constraints can be applied to select significant coefficients, such as thresholds on the modified $\chi^{2}$ value defined in Eq. 2 or any ancillary mask that isolates astrophysical contaminants. The heterogeneity of HFI beam widths is eventually taken into account via a frequency-dependent van Cittert [7] deconvolution of denoised frequency maps.

While this approach allowed us to restore anisotropic details such as shocks and filaments in the brightest regions of simulated clusters, the van Cittert deconvolution turned out to diverge for very faint values of the Compton parameter $\left(y<5 \times 10^{-6}\right)$, preventing us to recover features in the cluster peripheries. For this reason a new noniterative deconvolution technique has been proposed ([8], see also Baldi et al., these proceedings). Taking further advantage of the use of kernel-weighted likelihoods, we now compute wavelet coefficient as a two-step likelihood maximisation of dual scaling and wavelet functions. This allows us to recombine the negative and positive parts of the wavelet function, $\Psi$, within the coefficient likelihood, and to introduce a frequency-dependent convolution of their step-like supports, $H$, by the Planck beams $b_{v}$. For a spatial variation $\Delta y_{v}$ of the searched template $y_{v}$ encoded by each wavelet coefficient, deconvolved coefficient amplitudes are thus restored via:

$$
\begin{aligned}
& y^{\prime}(i, j, v)=b_{v}(i, j) *\left[(1-H(i, j)) \times\left(y_{v}-\Delta y_{v}\right)\right]-b_{v}(i, j) *\left[H(i, j) \times\left(y_{v}+\Delta y_{v}\right)\right] \\
& w \chi^{2}(k, l)=\sum_{i, j, v} \frac{|\Psi|(i, j)}{\sigma(i-k, j-l, v)^{2}}\left[I(i-k, j-l, v)-y^{\prime}(i-k, j-l, v)\right]^{2} .
\end{aligned}
$$

This coefficient-wise deconvolution technique turned out to be more robust than the van Cittert deconvolution for low values of the Compton parameter. It allowed us in particular to evidence sub-structures in the outskirts nearby clusters of Planck catalogue [8], or to detect bridges that connect components of nearby cluster systems. As an example, a map of the tSZ signal toward the cluster pair A3395 - A3391 is shown in Fig. 2. This maps exhibits a blob to the south of A3395, and a sharp bridge that connects A3395 to A3391. The blob might coincide with accreted galaxy group ESO-161 visible in X-ray from a composite Chandra observation (see details in [9]).

\section{All-sky mapping of the thermal SZ signal}

Looking for hot baryons outside known galaxy clusters motivates the development of full-sky spectral-imaging algorithms. We generalised cluster imaging algorithms illustrated in section 3 to B3-spline wavelets on the sphere. Similarly to the cartesian case, we implemented a fully parametric approach of spectral-fitting with four free parameters.

A half-sky map of the thermal-SZ signal smoothed with a spline kernel of width of about 10 arcminutes is shown in Fig. 3. Most North hemisphere clusters of the Planck catalogue are visible on this map, the most extended of them, such as the Coma cluster and the Shapley 


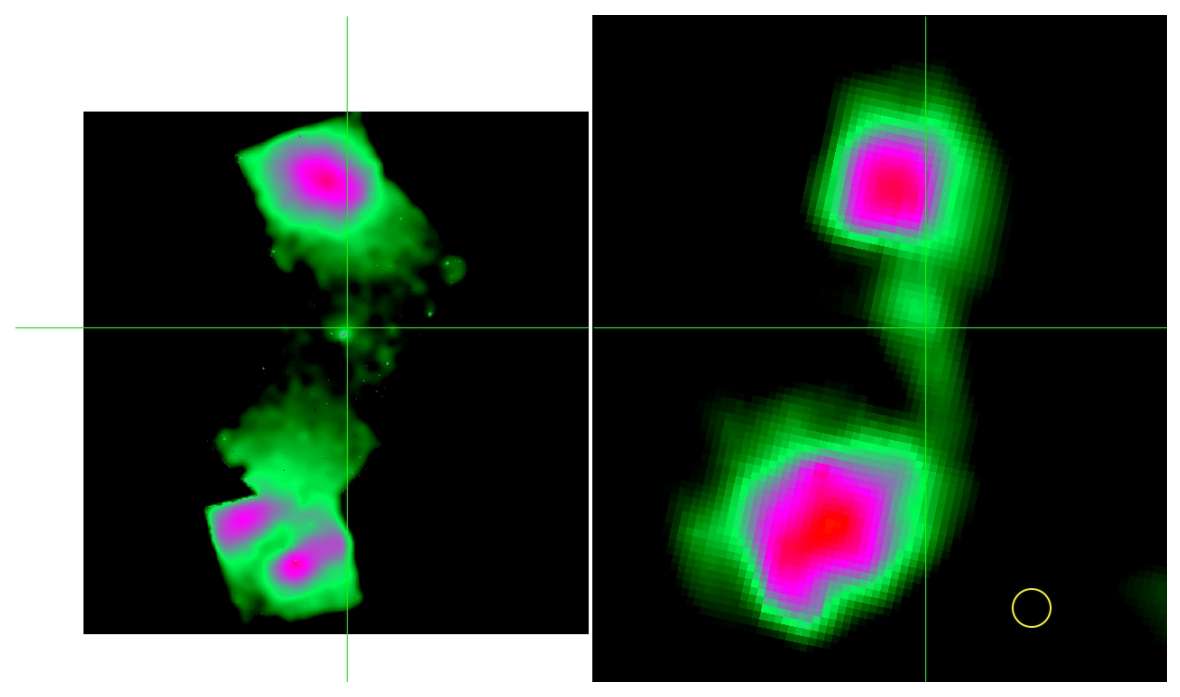

Figure 2. Cluster pair A3395-A3391 as seen by Chandra and Planck. Left panel: Wavelet denoised $\mathrm{X}$-ray surface brightness in the energy band $0.5-2.5 \mathrm{keV}$. Right panel: Curvelet denoised tSZ signal. Yellow circle represents a 5 arcmin width beam. Both the X-ray and the mm images evidence a blob to the South of A3395, that coincides with galaxy group ESO-161 (see [9] for details about Chandra observations of this system).

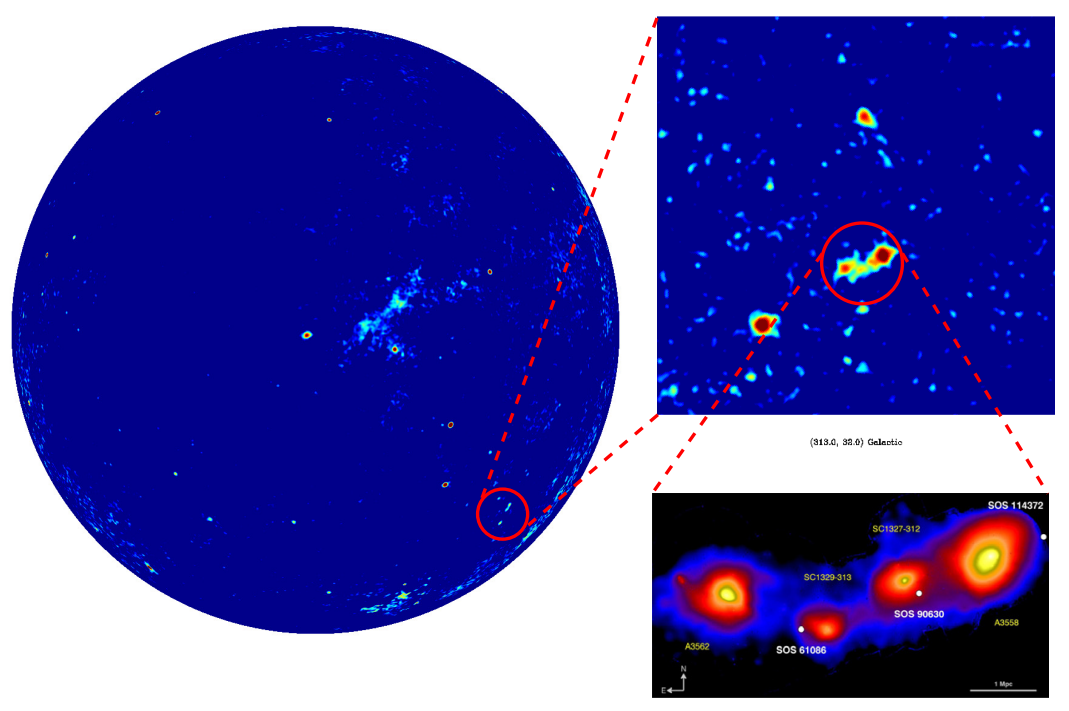

Figure 3. Left panel: Half-sky map (North Galactic hemisphere) of the thermal SZ smoothed with a spline kernel of typical width of 10 arcminutes. Top-right panel: Detail of the map in the neighbourhood of the Shapley Supercluster core. Bottom-right panel: Wavelet denoised X-ray surface brightness in the energy band $0.5-2.5 \mathrm{keV}$ (see [10] for details about XMM-Newton observations of this system). 
supercluster core, being spatially resolved with their characteristic shape. Interestingly the morphology of the Shapley Supercluster core exhibits four components whose alignment is similar to what is observable in X-rays (e.g. [10]). A residual contamination of the tSZ map by a Galactic cirrus is visible at high Galactic latitude in the North hemisphere. Independent wavelet analyses of the spatial variations of dust spectral index and tSZ signal should allow us to reduce the remaining degeneracy between these components, especially by exploiting our prior knowledge about differences in the typical spatial scales of these two components.

\section{Conclusion and perspectives}

We developed component separation algorithms that take advantage of wavelet and curvelet analyses to denoise and map astrophysical contaminants of the tSZ signal as detected by Planck HFI. Residuals of these contaminants around individual clusters or stacked cluster images show us that thermal dust contamination around high SNR clusters in the Planck catalogue can be modelled by using a three component grey-body, including two components for the Galactic emission and a third component for the cluster emission. A similar modelling of the SED of tSZ contaminants has been used to map the Compton parameter for nearby, spatially resolved clusters in the Planck catalogue. Specifically, spatially weighted likelihood allowed us to jointly fit wavelet and curvelet coefficients of the Compton parameter with coefficients of the contaminants. This model-driven approach permitted in particular to add non linear constraints to the noise-driven coefficient thresholding (e.g. $\chi^{2}$ limits or ancillary masks) and to introduce a coefficient-wise deconvolution procedure. This new deconvolution procedure turned out to be more stable than a state-of-the-art van Cittert deconvolution algorithms. We took advantage from this approach to detect substructures and filaments in the outskirts $\left(r>r_{500}\right)$ of several nearby clusters, including most members of the Planck SNR selected cluster sample of the X-ray Clusters Outskirts Project [8]. Combining HFI data with observations performed at higher angular resolutions (e.g. NIKA2) should allow us to best resolve features that populate the virialization region of nearby clusters. Such algorithms could also be generalised to all-sky mapping of the tSZ signal via the use of wavelet coefficients on the celestial sphere, with possible applications including the decontamination of foregrounds in the Planck CMB maps.

\section{References}

[1] Planck Collaboration, A\&A594, A27 (2016), 1502.01598

[2] H. Bourdin, P. Mazzotta, A. Kozmanyan, C. Jones, A. Vikhlinin, The Astrophysical Journal 843, 72 (2017), 1707.02248

[3] A.M. Meisner, D.P. Finkbeiner, ApJ798, 88 (2015), 1410.7523

[4] Planck Collaboration, A\&A596, A104 (2016), 1603.04919

[5] H. Bourdin, P. Mazzotta, E. Rasia, The Astrophysical Journal 815, 92 (2015), 1601.06323

[6] S. Mallat, A Wavelet Tour of Signal Processing (Academic Press, 1999)

[7] P.H. van Cittert, Zeitschrift für Physik 69, 298 (1931)

[8] A.S. Baldi, H. Bourdin, P. Mazzotta, D. Eckert, S. Ettori, M. Gaspari, M. Roncarelli, arXiv e-prints arXiv:1906.10013 (2019), 1906. 10013

[9] G.E. Alvarez, S.W. Randall, H. Bourdin, C. Jones, K. Holley-Bockelmann, ApJ858, 44 (2018), 1802.08688

[10] P. Merluzzi, G. Busarello, M.A. Dopita, C.P. Haines, D. Steinhauser, H. Bourdin, P. Mazzotta, MNRAS460, 3345 (2016), 1605.06329 